\title{
COVERING SPACES OF 3-MANIFOLDS
}

\author{
JOEL HASS, HYAM RUBINSTEIN AND PETER SCOTT
}

Let $M$ be a closed $P^{2}$-irreducible 3-manifold with infinite fundamental group. It is a long-standing conjecture that the universal cover $\tilde{M}$ of $M$ must be homeomorphic to $R^{3}$. Waldhausen [Wa] proved that this is the case when $M$ is Haken. The first result of this announcement is the following generalization of Waldhausen's result.

THEOREM 1. Let $M$ be a closed $P^{2}$-irreducible 3-manifold. If $\pi_{1}(M)$ contains the fundamental group of a closed surface other than $S^{2}$ or $P^{2}$ then the universal cover of $M$ is homeomorphic to $R^{3}$.

We will say that a 3-manifold is almost compact if it can be obtained from a compact manifold $N$ by removing a closed subset of $\partial N$. Then Theorem 1 is equivalent to the assertion that the universal covering of $M$ is almost compact. A natural way to attempt to generalize Theorem 1 is to show that other coverings of $M$ are almost compact. It was conjectured by Simon [Si] that if $M$ is any compact $P^{2}$-irreducible 3-manifold and if $M_{1}$ is a covering of $M$ with finitely generated fundamental group then $M_{1}$ must be almost compact. Simon verified this conjecture for the case when $\pi_{1}\left(M_{1}\right)$ is the fundamental group of a boundary component of $M$. Jaco $[\mathbf{J}]$ generalized this to the case when $\pi_{1}\left(M_{1}\right)$ is a finitely generated peripheral subgroup of $\pi_{1}(M)$. More recently, Thurston [Th] showed that if $M$ admits a geometrically finite complete hyperbolic structure of infinite volume then Simon's conjecture is true. Finally, Bonahon [B] showed that any hyperbolic 3-manifold with finitely generated fundamental group is almost compact provided that $\pi_{1}(M)$ is not a free product.

The second result of the announcement is the following.

THEOREM 2. Let $M$ be a closed $P^{2}$-irreducible 3-manifold such that $\pi_{1}(M)$ contains a subgroup $A$ isomorphic to $Z \times Z$. Then the covering $M_{A}$ of $M$ with $\pi_{1}\left(M_{A}\right)=A$ is almost compact.

If $M$ is Haken then the conclusion of the theorem follows immediately from Simon's work [Si]. For the crucial condition needed in [Si] is that if $H$ is any finitely generated subgroup of $\pi_{1}(M)$, then $H \cap A$ is also finitely generated, and this obviously holds, as $A$ is isomorphic to $Z \times Z$. If $M$ is not Haken, then the version of the Torus Theorem proved by Scott [Sc] shows that some infinite cyclic subgroup of $A$ must be normal in $\pi_{1}(M)$. Now the following is another long-standing conjecture.

Received by the editors September 4, 1986.

1980 Mathematics Subject Classification (1985 Revision). Primary 57M10.

(C) 1987 American Mathematical Society $0273-0979 / 87 \$ 1.00+\$ .25$ per page 
CONJECTURE 3. If $M$ is a closed $P^{2}$-irreducible 3-manifold such that $\pi_{1}(M)$ contains an infinite cyclic normal subgroup, then $M$ is a Seifert fiber space.

If $M$ is a Seifert fiber space, then Theorem 2 is easily proved. Thus the interest of Theorem 2 is that we do not assume that $M$ is either Haken or a Seifert fiber space. We hope that our result will be a step towards proving Conjecture 3.

Now we give a brief outline of the arguments for proving Theorem 1. Suppose that $F$ is a closed surface, not $S^{2}$ or $P^{2}$, such that $\pi_{1}(M)$ contains $\pi_{1}(F)$. Then there is a map $f: F \rightarrow M$ inducing the inclusion of fundamental groups and we choose $f$ to be a least-area map in its homotopy class. This can be done in the smooth category by choosing a Riemannian metric on $M$ and then applying the existence results of Schoen-Yau [S-Y], or it can be done in the P-L category using work of Jaco and Rubinstein [J-R]. Now in the universal cover $\tilde{M}$ of $M$, the complete pre-image $\Pi$ of $f(F)$ consists of planes as $f$ injects $\pi_{1}(F)$ into the fundamental group of $M$, and these planes are embedded by results of Freedman, Hass, and Scott [F-H-S]. Further, the intersection of two planes never contains a circle. These planes define a division of $M$ into 3-dimensional chambers, each of which covers one of the compact chambers in $M$ obtained by cutting $M$ along $f(F)$. We show that each of the chambers in $\tilde{M}$ is simply connected and that each chamber in $M$ is $\pi_{1}$-injective. We also show that if $M$ is not Haken then each chamber in $M$ is a handlebody. It follows that the closure of each chamber in $\tilde{M}$ is almost compact. We show that if a finite collection $\Sigma$ of the planes in $\Pi$ are removed, then the new bigger chambers formed by cutting $\tilde{M}$ along $\Pi-\Sigma$ have the same property. As a compact set in $\tilde{M}$ can meet only finitely many planes of $\Pi$, it follows immediately that any compact subset of $\tilde{M}$ lies in the interior of a 3-ball. This shows that $M$ is homeomorphic to $R^{3}$ as required.

The proof of Theorem 2 uses similar ideas. Let $T$ denote the 2-torus and let $f: T \rightarrow M$ be a map of least area such that $f_{*}\left(\pi_{1}(T)\right)=A$. The preimage in $M_{A}$ of $f(T)$ is a collection of possibly singular tori and annuli. If they were all embedded in $M_{A}$ then we would argue much as in the proof of Theorem 1. This need not be the case, even when $M$ is a Seifert fiber space, but we show that $M_{A}$ must have a finite cover in which the preimage of $f(T)$ consists of embedded tori and annuli. This result completes the proof of Theorem 2.

\section{REFERENCES}

[B] F. Bonahon, Bouts des variétés hyperboliques de dimension 3, Ann. of Math. (2) 124 (1986), 71-158.

[F-H-S] M. H. Freedman, J. Hass and P. Scott, Least area incompressible surfaces in 3-manifolds, Invent. Math. 71 (1983), 609-642.

[J] W. Jaco, Lectures on 3-manifold topology, CBMS Regional Conf. Ser. in Math., no. 43, Amer. Math. Soc., Providence, R.I., 1980.

[J-R] W. Jaco and H. Rubinstein, $P$ - $L$ minimal surfaces in 3-manifolds, preprint.

[Sc] P. Scott, A new proof of the annulus and torus theorems, Amer. J. Math. 102 (1980), 241-277. 
[Si] J. Simon, Compactification of covering spaces of compact 3-manifolds, Michigan Math. J. 23 (1976), 245-356.

[S-Y] R. Schoen and S. T. Yau, Existence of incompressible minimal surfaces and the topology of 3-dimensional manifolds with non-negative scalar curvature, Ann. of Math. (2) 110 (1974), 127-142.

[Th] W. Thurston, Geometry and topology of 3-manifolds, Lecture Notes, Princeton University, 1978-1979.

[Wa] F. Waldhausen, On irreducible 3-manifolds which are sufficiently large, Ann. of Math. (2) 87 (1968), 56-88.

Department OF MATHEMATICS, Hebrew UNiversity of JeruSALEM, JERUSALEM, ISRAEL

Department of Mathematics, Melbourne University, Parkville, VicTORIA 3052, AUSTRALIA

DePaRTMENT OF MATHEMATICS, UNIVERSity OF LiVerpool, LiVERPoOL, ENGLAND 
